\title{
Planning and monitoring of patients for electrical cardioversion for atrial fibrillation
}

\author{
J. H. H. Deuling • R. P. Vermeulen • M. D. Smit • \\ J. M. A. A. van der Maaten - H. M. Boersema • \\ A. F. M. van den Heuvel • I. C. Van Gelder
}

Published online: 29 October 2011

(C) The Author(s) 2011. This article is published with open access at Springerlink.com

\begin{abstract}
Objectives This study evaluated the waiting list for elective electrical cardioversion (ECV) for persistent atrial fibrillation (AF), focusing on when and why procedures were postponed. We compared the effects of management of the waiting list conducted by physicians versus management by nurse practitioners (NPs) and we evaluated the safety of our anticoagulating policy by means of bleeding or thromboembolic complications during and after ECV.

Background Not all patients selected for ECV receive their treatment at the first planned instance due to a variety of reasons. These reasons are still undocumented.

Methods We evaluated 250 consecutive patients with persistent AF admitted to our clinic for elective ECV.

Results Within 5 to 6 weeks, 186 of 242 patients (77\%) received ECV. The main reason for postponing an ECV was
\end{abstract}

J. H. H. Deuling $\cdot$ R. P. Vermeulen - M. D. Smit •

H. M. Boersema - A. F. M. van den Heuvel - I. C. Van Gelder

Department of Cardiology, University Medical Centre Groningen,

University of Groningen,

Groningen, the Netherlands

J. M. A. A. van der Maaten

Department of Anesthesiology, University Medical Centre

Groningen, University of Groningen,

Groningen, the Netherlands

I. C. Van Gelder

Interuniversity Cardiology Institute Netherlands,

Utrecht, the Netherlands

I. C. Van Gelder $(\bowtie)$

Thorax Centre, Department of Cardiology, University Medical

Centre Groningen, University of Groningen,

PO Box 30.001, 9701 RB Groningen, the Netherlands

e-mail: i.c.van.gelder@umcg.nl an inadequate international normalised ratio (INR); other reasons included spontaneous sinus rhythm and switch to rate control. A total of 23 of the 147 patients (16\%) managed by the research physician were postponed due to an inadequate INR at admission versus 4 out of 98 patients (4\%) managed by NPs $(p=0.005)$

Conclusion An inadequate INR is the main reason for postponing an ECV. Management of ECV by NPs is safe and leads to less postponing on admission.

Keywords Electrical cardioversion · INR monitoring · Nurse practitioner

\section{Introduction}

Atrial fibrillation (AF) is the most common arrhythmia in clinical practice. Rhythm and rate control are the two treatment strategies of patients with AF. For most cardiologists rhythm control is the treatment of choice in symptomatic AF patients $[1,2]$.

An electrical cardioversion (ECV) is one of the therapies for rhythm control in patients with persistent AF. Because of thromboembolic complications, an ECV procedure has to be performed within $48 \mathrm{~h}$ after onset of $\mathrm{AF}$ or electively after a minimum period of three weeks of adequate level of anticoagulation [3]. For determining adequate anticoagulation status, the international normalised ratio (INR) can be used in patients treated with vitamin $\mathrm{K}$ antagonists. Worldwide the international INR value should range between 2.0 and 3.0 for patients with AF $[3,4]$. To avoid undercoagulation in particular, the Dutch anticoagulation clinics use slightly higher therapeutic target ranges of the INR, namely between 2.5 and 3.5 . 
Not all patients selected for ECV receive their treatment at the first planned hospital admission due to a variety of reasons. The incidence and reasons for postponing ECV procedures are still not thoroughly studied. The aim of our study was therefore to evaluate the waiting list for elective ECV for patients with persistent AF in the Department of Cardiology of the University Medical Centre Groningen, the Netherlands
(UMCG), focusing on how often and for what reason procedures were postponed. Furthermore, we compared the effects of the management of the waiting list conducted by physicians versus management by nurse practitioners (NPs). Finally we evaluated the safety of our anticoagulation therapy and policy by means of bleeding or thromboembolic complications during and after ECV.
Fig. 1 Protocol for electrical cardioversion at the University Medical Centre Groningen

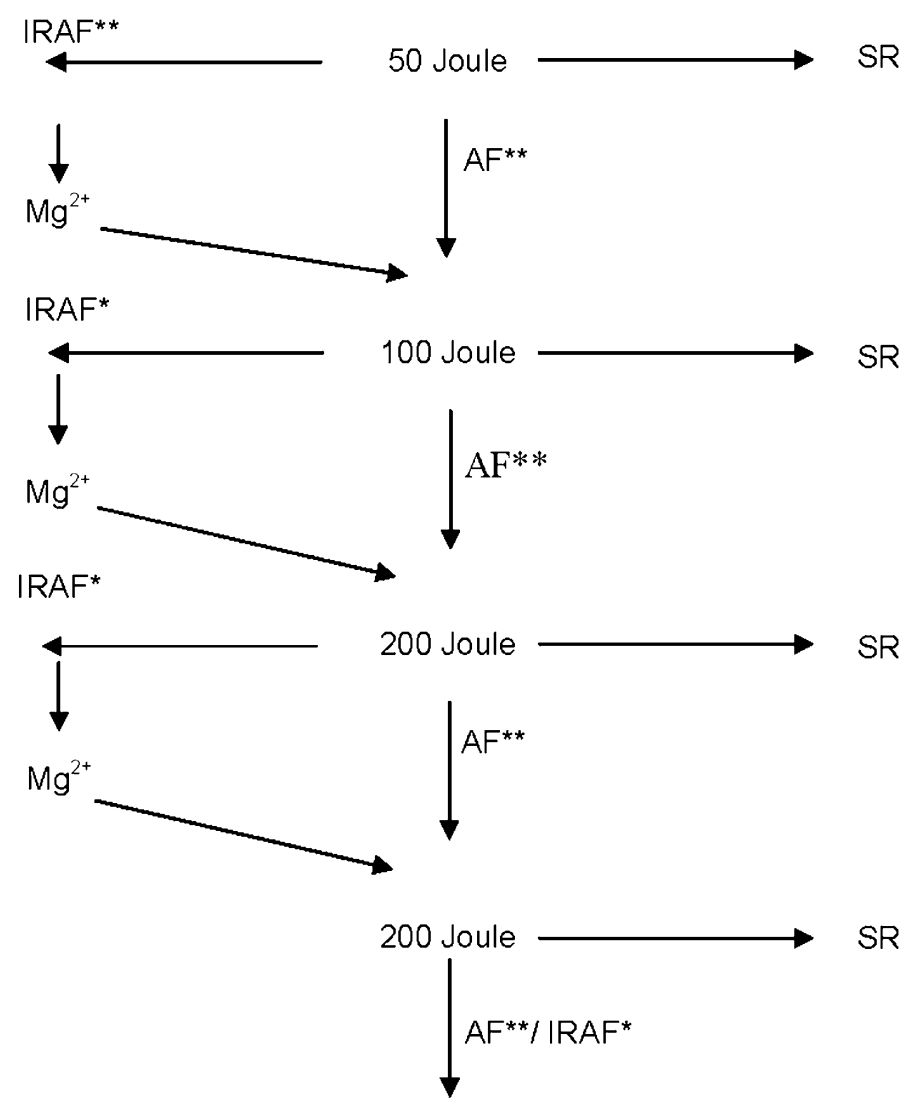

Verapamil intravenously ( $5 \mathrm{mg}$ in $5 \mathrm{~min}$ )

(only with adequate on blood pressure, heart rate and left ventricular function)

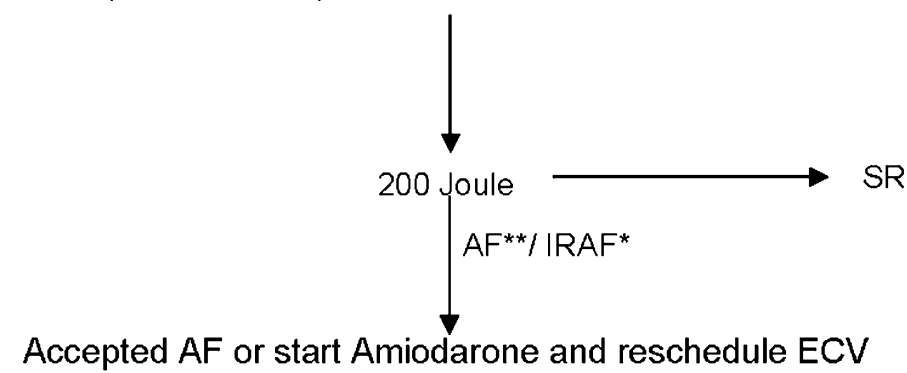

SR: Sinus rhythm

IRAF*: Immediate Reinitiation of $A F<2$ min after ECV.

$A F^{\star *}$ : Atrial fibrillation, no sinus beat observed after ECV 


\section{Methods}

Patients

This was a prospective observational study performed in the UMCG between August 2008 and December 2009. We evaluated 250 consecutive patients with persistent AF admitted to our clinic for elective ECV. The choice for a rhythm-control strategy was made by a dedicated AF cardiologist and cardiologist in training, based upon medical history, AF-associated symptoms, current medication and echocardiographic parameters, after identifying and treating underlying diseases which may play a role in onset and maintenance of AF. The INR status was monitored in the period before ECV by the physician or NP. If the patient's INR dropped below the therapeutic target range during the period between acceptance for ECV and performing ECV, the ECV was postponed or a transoesophageal echocardiography (TEE) was performed to exclude thrombus in the left atrium or left atrial appendage [5]. Additionally, if the INR was below the target range or above 5.5 on the day of admission, the ECV was cancelled and a new appointment was made for the patient. According to our hospital protocol, a minimum continuous period of 4 weeks of adequate anticoagulation was required.

The relevant medical management from acceptation until discharge after ECV was performed by the physician until 15 June 2009. After this date, the tasks shifted. The physician became responsible for selection of patients for $\mathrm{ECV}$, initial contact with the anticoagulation clinic and the initial planning on the waiting list, while the NP at the short stay unit was made responsible for maintaining contact with the anticoagulation clinic and the necessary mutations on the waiting list. The NP also started performing the ECV.

\section{Electrical cardioversion}

ECV was performed under short general anaesthesia provided by a consulting anaesthesiologist [6]. Anaesthesia was induced with propofol (typically $0.5-1 \mathrm{mg} / \mathrm{kg}$ ) until loss of consciousness. Additional bolus doses of propofol were administered for maintenance of anaesthesia during the procedure. Monitoring consisted of non-invasive blood pressure measurement and pulse oxymetry.

The ECV was performed according to a standardised protocol as has been described earlier (Fig. 1) [7]. In case of immediate recurrence of AF, $750 \mathrm{mg}$ magnesium was given to increase the likelihood of successful cardioversion and to maintain sinus rhythm afterwards [8]. A brief observation period after ECV to assess complete recovery was provided by the anaesthesia team. Patients were usually discharged from the hospital 2-4 h after ECV if they were well awake and in the absence of complications. In case of initiation of class I or III antiarrhythmic drugs, hospitalisation was prolonged for a period of $24-48 \mathrm{~h}$.

Endpoints and follow-up

Primary endpoints of this study were time from acceptance for ECV to date of the procedure, and reasons for postponing the procedure, compared between management by physician or NP. The secondary endpoints were bleeding or thromboembolic complications. Follow-up was performed after the first outpatient clinic visit by reviewing patient medical records.

\section{Statistical analysis}

All data were analysed using SPSS version 16.0 (SPSS, Illinois USA). Statistics are presented as mean \pm standard deviation (SD) or median (interquartile range Q25-Q75) for continuous variables, or as numbers with percentages for categorical variables.

We used the Mann-Whitney and $\chi^{2}$ tests to analyse the results. A double-sided $\mathrm{p}$ value of 0.05 or less was considered statistically significant.

Table 1 Baseline characteristics

\begin{tabular}{ll}
\hline Characteristic & All patients $(n=242)$ \\
\hline Age (years) & $63 \pm 11$ \\
Male sex & $173(71.5)$ \\
BMI $\left(\mathrm{kg} / \mathrm{m}^{2}\right)$ & $27 \pm 4$ \\
Current AF duration (months) & $3(1-15)$ \\
Previous electrical cardioversion & $2(1-3)$ \\
Hypertension & $126(52.1)$ \\
Coronary artery disease & $55(22.7)$ \\
Valvular heart disease & $14(5.8)$ \\
COPD & $6(2.5)$ \\
Diabetes & $23(9.5)$ \\
History of heart failure & $53(21.9)$ \\
CHA ${ }_{2}$ DS \\
2
\end{tabular}

Data are expressed as number $(\%)$ or mean $\pm \mathrm{SD}$ or median (interquartile range)

$A A D$ antiarrhythmic drug; $B M I$ body mass index; $C O P D$ chronic obstructive pulmonary disease; NYMA New York Heart Association 


\section{Results}

First planned ECV

Of the 250 patients referred for electrical cardioversion, 8 patients $(3 \%)$ were primarily not accepted for an ECV because of an inappropriate treatment of underlying diseases. Table 1 shows baseline characteristics of the 242 patients who underwent ECV. Most were male (72\%) with a mean age of 63 years. Duration of the current AF episode was a median of 3 months. Figure 2 shows the flowchart of patients who were scheduled for ECV. Within 5 to 6 weeks, 186 of 242 patients $(77 \%)$ received ECV. After 5 rescheduled ECVs, all patients on the waiting list had been treated with $\mathrm{ECV}(n=218,90 \%)$. Patients were taken off the waiting list for reasons of spontaneous conversion to sinus rhythm $(n=16,7 \%)$, switch to rate control $(n=5,2 \%)$, hospital admission for symptomatic AF necessitating an
Fig. 2 Patient flow chart over planned and rescheduled ECV procedures

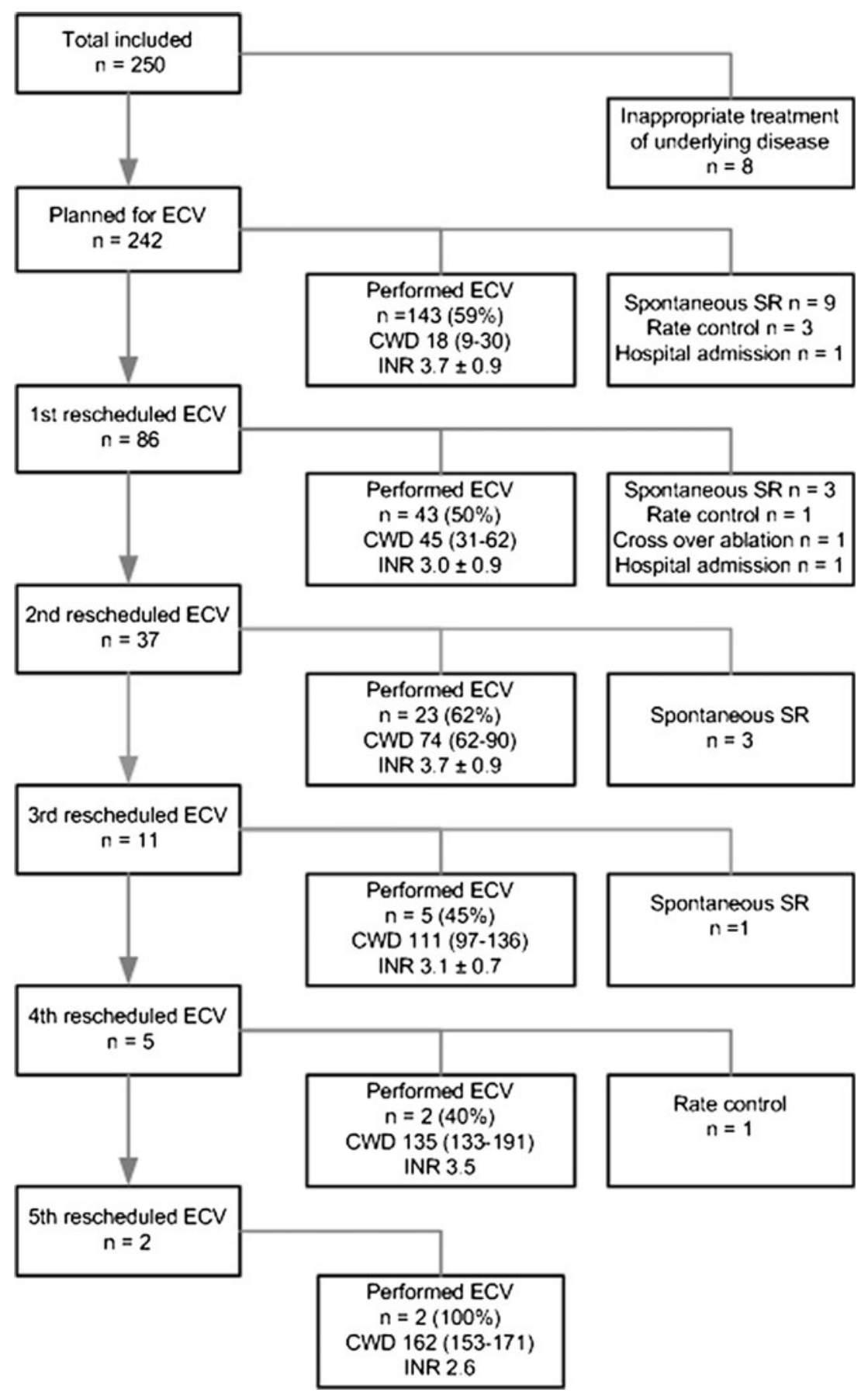


Table 2 Logistic parameters for electrical cardioversion

\begin{tabular}{lll}
\hline & $\begin{array}{l}\text { Postponed before } \\
\text { admission }\end{array}$ & $\begin{array}{l}\text { Postponed on } \\
\text { admission }\end{array}$ \\
\hline $\begin{array}{l}\text { First planned ECV } \\
n=242\end{array}$ & $65(27)$ & $34(14)$ \\
$\begin{array}{l}\text { Rescheduled I } \\
n=86\end{array}$ & $28(33)$ & $15(17)$ \\
$\begin{array}{l}\text { Rescheduled II } \\
n=37\end{array}$ & $11(30)$ & $3(8)$ \\
$\begin{array}{l}\text { Rescheduled III } \\
n=11\end{array}$ & $5(45)$ & $1(9)$ \\
$\begin{array}{l}\text { Rescheduled IV } \\
n=5\end{array}$ & $2(40)$ & $1(20)$ \\
$\begin{array}{l}\text { Rescheduled V } \\
n=2\end{array}$ & 0 & 0 \\
\hline
\end{tabular}

Data are expressed as number of eligible patients (\%)

unplanned ECV $(n=1,1 \%)$, sustained ventricular tachycardia $(n=1,1 \%)$, and crossover to pulmonary vein ablation $(n=1,1 \%)$.

The initially planned ECV was postponed in 99 (41\%) patients (Table 2). Reasons to postpone the ECV before admission $(n=65)$ were inadequate INR, patients' personal circumstances, switch to rate control, spontaneous conversion to sinus rhythm and unplanned hospital admission. Reasons to postpone the ECV on admission $(n=34)$ were INR under the therapeutic range ( $n=11$, mean INR $1.9 \pm 0.2)$, INR above the therapeutic range $(n=8$, mean INR $7.2 \pm 1.2)$, sinus rhythm at presentation, and other reasons, such as hyperkalaemia or bradycardia. Seven patients underwent
TEE before ECV; two procedures were postponed because of the presence of a thrombus in the left atrial appendage. Figure 3 displays the percentages of patients whose procedure was postponed at the first planned and each consecutive rescheduled ECV, before and on admission.

First rescheduled ECV

Eighty-six patients (36\%) were planned for a first rescheduled ECV, which was postponed again in 43 patients $(50 \%)$. Reasons to postpone before admission $(n=28)$ were inadequate INR, patients' personal circumstances and unscheduled hospital admission. Reasons to postpone on admission day $(n=15)$ were INR under the therapeutic range ( $n=5$, mean INR $1.8 \pm 0.5)$, INR above the therapeutic range $(n=1, \mathrm{INR}=7.0)$, sinus rhythm, and other reasons such as bradycardia and poor adherence to the medical regimen. Four patients underwent TEE before ECV; in three patients this was postponed because of thrombus in the left atrial appendage.

\section{Second and further rescheduled ECVs}

Thirty-seven patients $(15 \%)$ were planned for a second rescheduled ECV. This was postponed for the second time in 14 patients (38\%). Reasons to postpone ECV before admission $(n=11)$ were inadequate INR, spontaneous sinus rhythm and haematuria. Reasons to postpone the ECV on admission $(n=3)$ were signs of heart failure and sinus rhythm.

Eleven patients $(5 \%)$ were planned for a third rescheduled ECV. In 6 patients (55\%) this was postponed for the third time.
Fig. 3 Reasons for postponing ECV

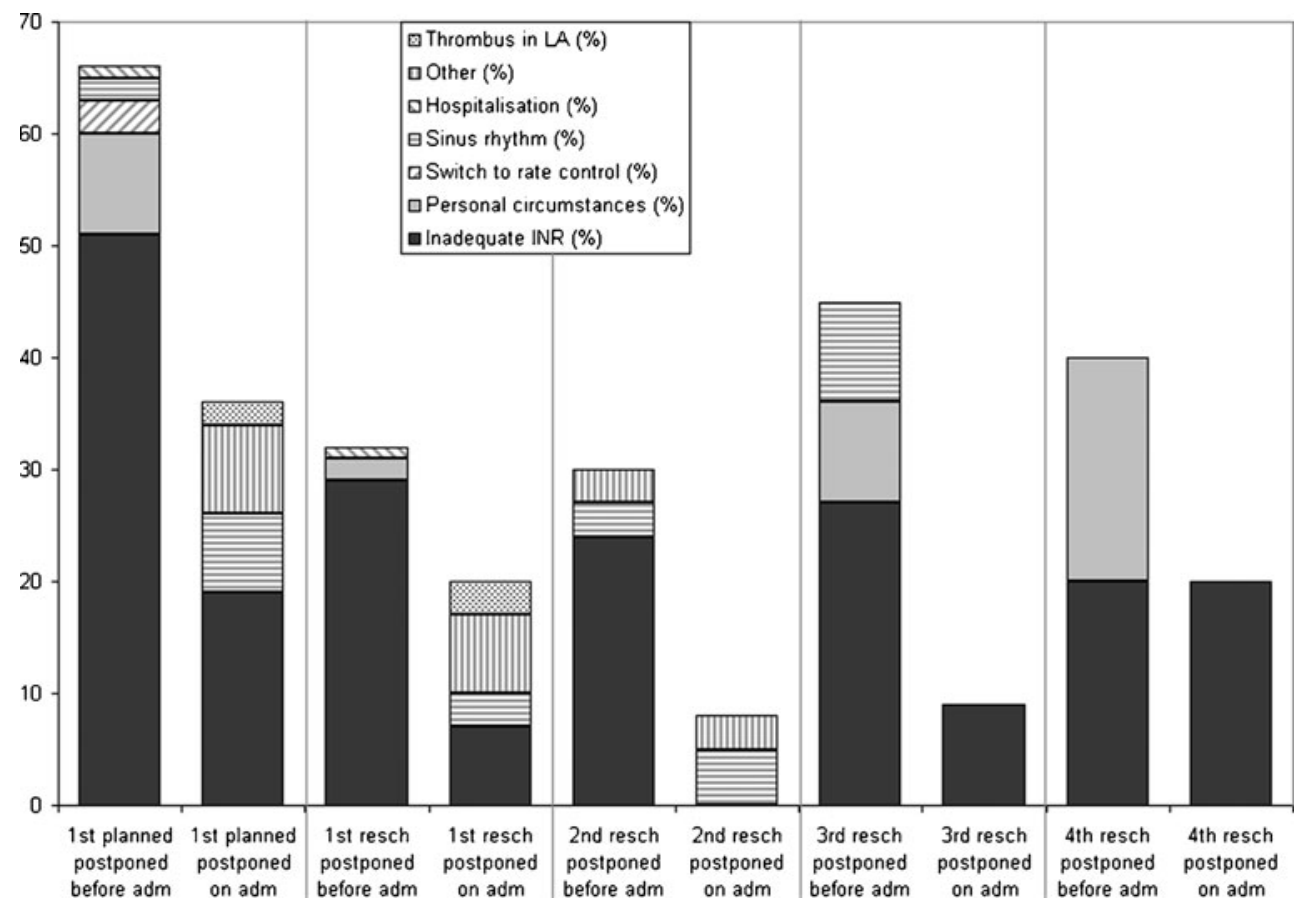


Reasons to postpone ECV before planning $(n=5)$ were inadequate INR, patients' personal circumstances and spontaneous sinus rhythm. The reason to postpone the ECV on admission $(n=1)$ was an INR under the therapeutic range $(\mathrm{INR}=2.0)$.

Five patients $(2 \%)$ were planned for a fourth rescheduled ECV. The procedure was postponed for the fourth time in three patients $(60 \%)$. Reasons to postpone ECV before admission $(n=2)$ were inadequate INR and patients' personal circumstances. The reason to postpone the ECV on admission $(n=1)$ was an INR over the therapeutic range $(\mathrm{INR}=$ $10.0)$. Two patients (1\%) were planned for a fifth rescheduled ECV. Both patients underwent this ECV as planned

Physician compared with dedicated NP

Over the course of all ECVs, the majority of the repeatedly postponed procedures were due to an inadequate INR (78\%). In total, patients were scheduled for an ECV 272 times. The procedure was postponed in 23 of the 147 patients (16\%) managed by the research physician due to an inadequate INR at admission versus 4 out of 98 patients (4\%) managed by the NP $(p=0.005)$. Analysis of waiting lists showed a similar number of cumulated waiting days before ECV between management by physician and NP (Fig. 4).

\section{Follow-up}

With regard to bleeding or thromboembolic complications, none of the 242 patients reported an event at the first outpatient clinic visit.

\section{Discussion}

The present study provides valuable information about patient and logistic parameters for planned ECVs. Our main finding was that ultimately $90 \%$ of selected patients

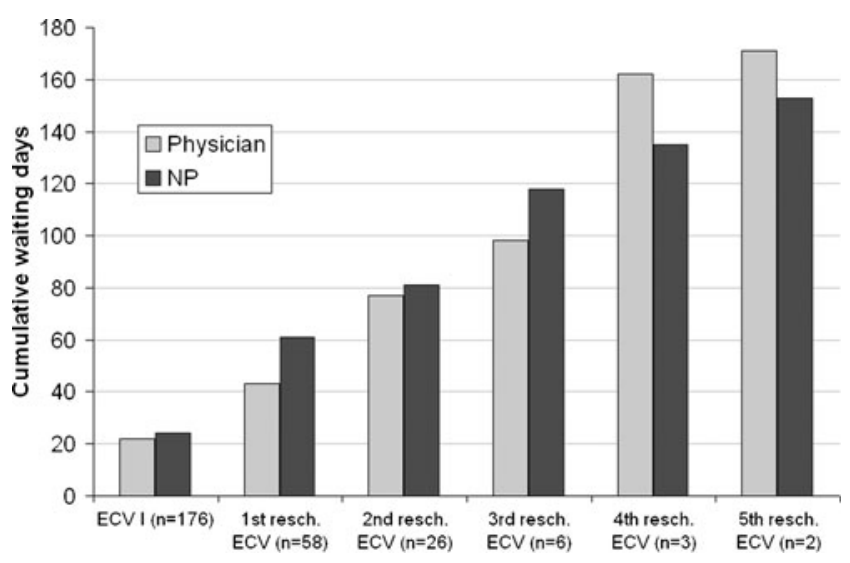

Fig. 4 Waiting days before ECV, managed by physician and NP received an ECV, despite some procedures being rescheduled 5 times. The main reason to postpone a planned ECV was an inadequate INR. In 7\% of patients, spontaneous conversion to sinus rhythm occurred. Furthermore, our study showed that in the patient group managed by an NP, the INR was more often adequate, leading to significantly less postponed ECVs at admission. Finally, in our patient group there were no bleeding or thromboembolic complications between ECV and first outpatient visit.

On the topic of anticoagulation status a number of papers have been published. In a clinical trial, a recent study showed that the patients included in the Netherlands were within the therapeutic range for $70 \%$ of the time [9]. However, in our study, which represents routine clinical care, we found a lower percentage of adequate INR values. Veeger et al. [10] reported a similar finding showing that in patients with $\mathrm{AF}$ the INR was within the target range during $42 \%$ of the time. When analysing the results, we did not discriminate between patients followed by the anticoagulation clinic and selftesting patients at home. A recent study shows that patients who perform self-testing have a small but significantly higher in-target INR range time than patients tested in a clinic. This, however, does not lead to a difference in thromboembolic or bleeding complications [11].

With the advance of dabigatran as anticoagulation medication instead of coumarin derivatives, closely following the INR status will become obsolete when planning patients for ECV. Dabigatran has been shown to be as effective and safe as warfarin in patients with $\mathrm{AF}$ with respect to incidence of stroke and systemic embolism, and lower or similar rates of major haemorrhage depending on the dosage. We expect that in the near future the number of postponed ECV procedures will decrease since INR status will no longer play a key role [9, 12-15]. On the other hand, no control on the intake of dabigatran may have deleterious effects when ECV is performed under inadequate anticoagulation in patients with low compliance and warrants attention.

A total of 16 patients (7\%) spontaneously converted to sinus rhythm in the period between acceptance and admission for ECV. Channer et al. reported spontaneous conversion to SR in $21 \%$ of patients while waiting for ECV [12]. All of these patients were treated with amiodarone $400 \mathrm{mg}$ twice daily. Tieleman et al. found similar results in patients with concomitant treatment with verapamil [16]. In our study 7 patients used amiodarone, 3 patients had a medication switch before ECV, 2 patients recently had an invasive pulmonary vein ablation, and 4 patients had SR without an identifiable cause.

Role of the NP

On our short stay unit, NPs systematically contact the anticoagulation clinics about the INR status of planned 
patients. Though this systematic check of INR may lead to increased postponing of procedures prior to hospital admission, it may also prevent patient discomfort, since fewer patients come to the hospital with an inadequate INR. The number of postponed procedures after the patient was admitted to the hospital showed a marked decrease under NP management. It is unclear why the total amount of waiting days did not decrease accordingly. Although this was not the subject of our study, we hypothesise that physicians checked INR less frequently due to other priorities. The feasibility and safety of nurse-led elective ECV procedures and subsequent chronic care programs with high guideline adherence have been reported earlier [17, 18].

The fact that we saw no thromboembolic complications at the first outpatient visit suggests that the approach of ECV management used is safe, a finding that has been reported previously [7].

Our study has some limitations. Firstly, it is a singlecentre study. Secondly, the number of patients decreased with each step, leaving a small number of patients in the fourth and fifth rescheduled ECV groups. This limits the generalisibility of these specific findings.

\section{Conclusion}

The main reason to postpone an ECV before and at admission is an inadequate INR. ECV was less often postponed on the day of admission in patients managed by the NP. Within 6 weeks from acceptance, $77 \%$ of patients received ECV. Sixteen patients developed sinus rhythm while waiting for ECV. Management on basis of INR value is safe.

Open Access This article is distributed under the terms of the Creative Commons Attribution Noncommercial License which permits any noncommercial use, distribution, and reproduction in any medium, provided the original author(s) and source are credited.

\section{References}

1. Van Gelder IC, Hagens VE, Bosker HA, et al. A comparison of rate control and rhythm control in patients with recurrent persistent atrial fibrillation. N Engl J Med. 2002;347(23):1834-40.

2. Wyse DG, Waldo AL, DiMarco JP, et al. A comparison of rate control and rhythm control in patients with atrial fibrillation. $\mathrm{N}$ Engl J Med. 2002;347(23):1825-33.

3. European Heart Rhythm Association, European Association for Cardio-Thoracic Surgery, Camm AJ, Kirchhof P, et al. Guidelines for the management of atrial fibrillation: the Task Force for the Management of Atrial Fibrillation of the European Society of Cardiology (ESC). Eur Heart J. 2010;31(19):2369-429.

4. Hirsh J, Fuster V, Ansell J, et al. American Heart Association/ American College of Cardiology Foundation. American Heart Association/American College of Cardiology. Foundation guide to warfarin therapy. J Am Coll Cardiol. 2003;41(9):1633-52.

5. European Heart Rhythm Association, European Association for Cardio-Thoracic Surgery, Camm AJ, Kirchhof P, et al. Guidelines for the management of atrial fibrillation: the Task Force for the Management of Atrial Fibrillation of the European Society of Cardiology (ESC). Europace. 2010;12(10):1360-420.

6. Van Gelder IC, Crijns HJ, Van Gilst WH, et al. Prediction of uneventful cardioversion and maintenance of sinus rhythm from direct-current electrical cardioversion of chronic atrial fibrillation and flutter. Am J Cardiol. 1991;68(1):41-6.

7. Van Gelder IC, Crijns HJ, Tieleman RG, et al. Chronic atrial fibrillation. Success of serial cardioversion therapy and safety of oral anticoagulation. Arch Intern Med. 1996;156(22):2585-92.

8. Davey MJ, Teubner D. A randomized controlled trial of magnesium sulfate, in addition to usual care, for rate control in atrial fibrillation. Ann Emerg Med. 2005;45(4):347-53.

9. Wallentin L, Yusuf S, Ezekowitz MD, et al. Efficacy and safety of dabigatran compared with warfarin at different levels of international normalised ratio control for stroke prevention in atrial fibrillation: an analysis of the RE-LY trial. Lancet. 2010;376 (9745):975-83.

10. Veeger NJ, Piersma-Wichers M, Tijssen JG, et al. Individual time within target range in patients treated with vitamin $\mathrm{K}$ antagonists: main determinant of quality of anticoagulation and predictor of clinical outcome. A retrospective study of 2300 consecutive patients with venous thromboembolism. $\mathrm{Br} \mathrm{J}$ Haematol. 2005;128(4):513-9.

11. Matchar DB, Jacobson A, Dolor R, et al. Effect of home testing of international normalized ratio on clinical events. N Engl J Med. 2010;363(17):1608-20.

12. Channer KS, Birchall A, Steeds RP, et al. A randomized placebo-controlled trial of pre-treatment and short- or longterm maintenance therapy with amiodarone supporting DC cardioversion for persistent atrial fibrillation. Eur Heart J. 2004;25(2):144-50.

13. Connolly SJ, Ezekowitz MD, Yusuf S, et al. Dabigatran versus warfarin in patients with atrial fibrillation. $\mathrm{N}$ Engl $\mathrm{J}$ Med. 2009;361(12):1139-51.

14. Nagarakanti R, Ezekowitz MD, Oldgren J, et al. Dabigatran versus warfarin in patients with atrial fibrillation: an analysis of patients undergoing cardioversion. Circulation. 2011;123(2):1316.

15. Verheugt FW. The new oral anticoagulants. Neth Heart J. 2010;18 (6):314-8.

16. Tieleman RG, Gosselink AT, Crijns HJ, et al. Efficacy, safety, and determinants of conversion of atrial fibrillation and flutter with oral amiodarone. Am J Cardiol. 1997;79(1):53-7.

17. Hendriks JL, Nieuwlaat R, Vrijhoef HJ, et al. Improving guideline adherence in the treatment of atrial fibrillation by implementing an integrated chronic care program. Neth Heart J. 2010;18(10):4717.

18. Shelton RJ, Allinson A, Johnson T, et al. Four years experience of a nurse-led elective cardioversion service within a district general hospital setting. Europace. 2006;8(1):81-5. 